\title{
Clinical utility of liquid biopsy for EGFR driver, T790M mutation and EGFR amplification in plasma in patients with acquired resistance to afatinib
}

Yuko Oya ${ }^{1}$, Tatsuya Yoshida ${ }^{1,2^{*}}$ (D, Kazuhiro Asada ${ }^{3}$, Tetsuya Oguri ${ }^{4}$, Naoki Inui ${ }^{5}$, Sayako Morikawa ${ }^{6}$, Kentaro Ito ${ }^{7}$, Tomoki Kimura ${ }^{8}$, Eiji Kunii ${ }^{9}$, Takashi Matsui ${ }^{10}$, Akihito Kubo ${ }^{11}$, Tatsuo Kato ${ }^{12}$, Takashi Abe ${ }^{13}$, Takeshi Tsuda ${ }^{14}$ and Toyoaki Hida'

\begin{abstract}
Background: Cell-free DNA (cfDNA) genotyping in plasma using the cobas EGFR Mutation Test v2 (cobas) is the first liquid biopsy as a companion diagnosis to identify the EGFR T790M mutation (T790M) after the failure of treatment of EGFR-tyrosine kinase inhibitors (TKIs) (1st generation, gefitinib [G] and erlotinib [E] and 2nd generation, afatinib [A]). This study investigated the clinical utility of a liquid biopsy for patients who acquired resistance to afatinib.

Methods: We prospectively collected plasma from 51 patients who had acquired resistance to afatinib between April 2015 and November 2016 to evaluate the frequency of T790M by cobas and digital droplet PCR (UMIN000025112). Additionally, we retrospectively reviewed 38 patients who tested by cobas in plasma after $\mathrm{G} / \mathrm{E}$ failure to compare for T790M detection between $A$ and with $\mathrm{G} / \mathrm{E}$.

Results: The detection rate of EGFR-driver and T790M in plasma in patients treated with A (A group) as a first-line EGFR-TKI was lower than with $\mathrm{G} / \mathrm{E}$ followed by $\mathrm{A}(\mathrm{G} / \mathrm{E} \rightarrow \mathrm{A}$ group), although the differences were not significant (EGFRdriver: $41 \%[\mathrm{~A}]$ vs. $67 \%[\mathrm{G} / \mathrm{E} \rightarrow \mathrm{A}], P=0.1867$; and T790M: $8 \%[\mathrm{~A}]$ vs. $17 \%[\mathrm{G} / \mathrm{E} \rightarrow \mathrm{A}], P=0.5798)$. In first-line setting, the detection rate for EGFR-driver and T790M in plasma by cobas was lower in A group than in G/E group, although there was no significant difference (EGFR-driver: 34\% [A] vs. 52\% [G/E], $P=0.2072$; and T790M: 10\% [A] vs. 27\% [G/E], $P=0.1161$ ).

Conclusion: The detection of EGFR-driver and T790M in plasma by cobas in patients treated with afatinib might be lower than with $G / E$ in a real-world setting.
\end{abstract}

Keywords: Epidermal growth factor receptor (EGFR), EGFR-tyrosine kinase inhibitor, Non-small cell lung cancer (NSCLC), Afatinib

\footnotetext{
* Correspondence: tatyoshi@ncc.go.jp

'Department of Thoracic Oncology, Aichi Cancer Center Hospital, 1-1

Kanokoden, Chikusa-ku, Nagoya, Aichi 464-8681, Japan

${ }^{2}$ Current Address: Department of Thoracic Oncology, National Cancer Center

Hospital, 5-1-1 Tsukiji, Chuo-ku, Tokyo 104-0045, Japan

Full list of author information is available at the end of the article
}

(c) The Author(s). 2021 Open Access This article is licensed under a Creative Commons Attribution 4.0 International License, which permits use, sharing, adaptation, distribution and reproduction in any medium or format, as long as you give appropriate credit to the original author(s) and the source, provide a link to the Creative Commons licence, and indicate if changes were made. The images or other third party material in this article are included in the article's Creative Commons licence, unless indicated otherwise in a credit line to the material. If material is not included in the article's Creative Commons licence and your intended use is not permitted by statutory regulation or exceeds the permitted use, you will need to obtain permission directly from the copyright holder. To view a copy of this licence, visit http://creativecommons.org/licenses/by/4.0/ The Creative Commons Public Domain Dedication waiver (http://creativecommons.org/publicdomain/zero/1.0/) applies to the data made available in this article, unless otherwise stated in a credit line to the data. 


\section{Background}

Epidermal growth factor receptor (EGFR) tyrosine kinase inhibitors (TKIs) has prolonged progression-free survival (PFS) compared with platinum-doublet chemotherapy as initial systemic therapy, and have been a standard firstline therapy for patients with EGFR mutations [1-3]. First-generation EGFR-TKIs, gefitinib and erlotinib, reversibly bind to and inhibit EGFR signaling, while second-generation EGFR-TKIs, such as afatinib and dacomitinib, irreversibly block the signaling from all relevant homo-dimers and hetero-dimers of the ErbB family receptors. Second-generation EGFR-TKIs have been reported to show a significantly longer PFS than first-generation EGFR-TKIs [4, 5].

The emergence of the EGFR T790M point mutation is the most common mechanism of acquired resistance to the EGFR-TKIs gefitinib, erlotinib and afatinib [6, 7]. Osimertinib, a third-generation and irreversible mutantselective EGFR-TKI, has been approved for advanced NSCLC patients harboring EGFR mutations, including the T790M mutation, based on the results of the AURA3 trial [8]. On the other hand, EGFR wild-type amplification has also been reported as a mechanism or resistance to EGFR-TKIs, including osimertinib $[9,10]$.

Cell-free DNA (cfDNA) genotyping in plasma using the cobas EGFR Mutation Test v2 (cobas test) is the first liquid biopsy to be approved as a companion diagnostic test to identify patients with the EGFR T790M mutation. cfDNA genotyping in plasma is a more easily accessible method of detecting T790M mutation than tissue-based biopsies. However, the AURA3 trial observed that only $51.2 \%$ of T790M-positive patients as evaluated using tumor tissues had T790M mutation as assessed using cfDNA in plasma [8], implying that the sensitivity of the cfDNA assay was insufficient to identify all T790M mutant-positive patients. Nevertheless, few reports have investigated the clinical utility of a liquid biopsy for detecting T790M mutation in patients with acquired resistance to afatinib, since most patients enrolled in the AURA3 trial were treated with gefitinib or erlotinib.

Therefore, we planned to investigate the clinical utility of a liquid biopsy for detecting T790M mutation in EGFRmutated NSCLC patients with acquired resistance to afatinib. In addition, we evaluated the difference in the detection of T790M in plasma from patients treated with firstgeneration EGFR-TKIs, and an EGFR wild-type amplification status in patients with acquired resistance to afatinib.

\section{Methods}

\section{Patients}

We studied two patient populations: a study arm consisting of patients enrolled in a prospective observational study, and a control arm consisting of patients in a retrospective study. For the study arm, we prospectively collected plasma samples from 51 patients who had been treated with afatinib, and had experienced progression during afatinib treatment between April 2015 and November 2016 at 13 institutions (Fig. 1a). The inclusion criteria were as follows: 1) a diagnosis of NSCLC, 2) a diagnosis of EGFR mutation, 3) the presence of progressive disease (PD) as assessed using the RECIST criteria, and 4) treatment with afatinib as the last EGFR-TKI to be administered prior to PD. Patients who had been treated with gefitinib/erlotinib (G/E) as the last EGFRTKIs before RECIST-PD were excluded. The presence of EGFR driver and/or T790M mutation was evaluated using the cobas test and digital droplet PCR (ddPCR).

In addition, to evaluate the difference in the detection of EGFR-driver and T790M mutation in plasma from patients treated with first-generation EGFR-TKIs (G/E) and afatinib as initia EGFR-TKIs, we retrospectively collected data on 33 patients who had been treated with G/ $\mathrm{E}$ as first-line EGFR-TKIs setting, and whose plasma samples had been analyzed using the cobas test to evaluate the presence of T790M mutation; these patients were regarded as a control arm (Fig. 1b). Moreover, if sufficient cfDNA samples were available after cobas testing and ddPCR for the T790M mutation, EGFR copy number variation $(\mathrm{CNV})$ was evaluated in an exploratory manner using ddPCR.

In all the patients, the patient characteristics, efficacy of EGFR-TKIs, and the T790M status in plasma, objective response rate (ORR), and PFS after treatment with EGFR-TKIs were reviewed. The protocol for this study was approved by the institutional review board of each institution, and the study was registered as a clinical trial (Clinical trial information: UMIN000025112).

\section{EGFR mutation and wild-type CNV assay in plasma}

To assess the presence of EGFR mutation (EGFR driver and T790M mutation) in plasma, approximately $20 \mathrm{~mL}$ of whole blood was collected using K2-EDTA collection tubes; within $4 \mathrm{~h}$ after blood collection, the samples were then centrifuged to separate the plasma from the peripheral blood cells at $1500 \times g$ for $10 \mathrm{~min}$ at $4{ }^{\circ} \mathrm{C}$, and the plasma supernatant was transferred to conical tubes and stored at $-80^{\circ} \mathrm{C}$ until transport. The plasma samples were transported at $-80^{\circ} \mathrm{C}$ to one of two commercial laboratories (SRL Inc., Tokyo, Japan, and G\&G Science Inc., Fukushima, Japan). The cobas EGFR Mutation Test v2 (Roche Molecular Systems, CA, USA) was used to detect EGFR mutations in the extracted plasma cfDNA. The mutant allele frequency and EGFR copy number were measured using the QX200 Droplet Digital PCR System (Bio-Rad, Hercules, CA). Isolated cfDNA was amplified using ddPCR Supermix for Probes (Bio-Rad) using EGFR T790M/L858R (PrimePCR ddPCR Mutation Assay; Bio-Rad), EGFR del19 assay (TaqMan Mutation 
A.

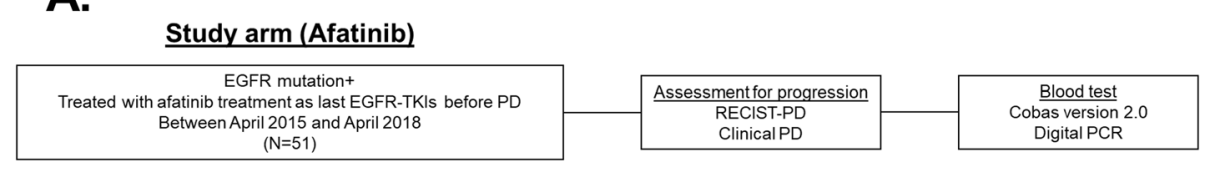

B.

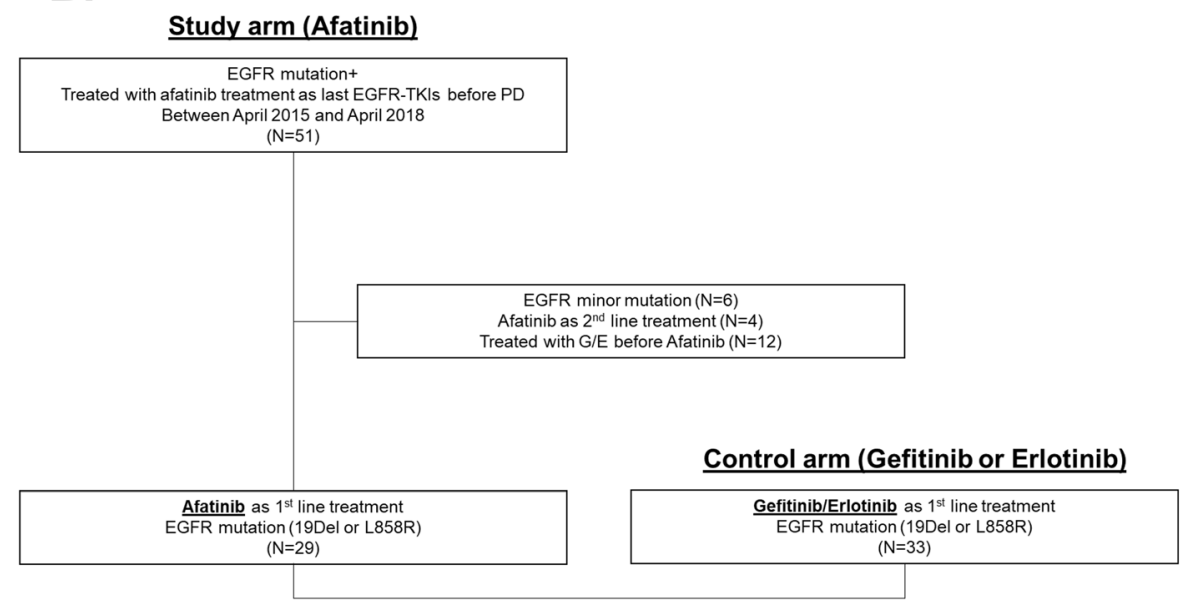

Fig. 1 Study schema. a A prospective observational study in which plasma samples were collected from patients with acquired resistance to afatinib ( $N=51$ ). b A comparison study of detection of EGFR driver and T790M mutation in plasm as assessed using the cobas test in between patients treated with afatinib as first-line setting enrolled in a prospective observation study and patients treated with $\mathrm{G} / \mathrm{E}$ as first-line setting in a retrospective study $(\mathrm{N}=62)$

Detection Assay; Thermo Fisher Scientific) for del19 EGFR mutations, and EGFR (RPP30 reference gene) for gene CNV (PrimePCR ddPCR Copy Number Assay; BioRad), according to the manufacturers' protocols. The ddPCR data were analyzed using Quanta Soft analytical software (version 1.7.4, Bio-Rad).

\section{Statistical analysis}

All the statistical analyses were performed using the JMP version 11 statistical software package (SAS Institute, Cary, NC, USA). Differences in the baseline characteristics between the groups were compared using the Fisher exact tests for categorical data. The response of treatment was evaluated based on Response Evaluation Criteria in Solid Tumors (RECIST version 1.1) [11]. The PFS was calculated from the date of therapy initiation to disease progression. The survival probabilities were estimated using the Kaplan-Meier method, where differences in the variables were calculated using the log-rank test. The cut-off date was August 30, 2018.

\section{Results}

\section{Patient characteristics}

Between April 2015 and November 2016, a total of 51 patients were enrolled in a prospective observational study (Fig. 1a). The characteristics of the patients are summarized in Table 1 . The median age of the patients was 67 (range, 36-81) years. Twenty-eight (55\%) were male. All the patients had adenocarcinoma, although the selection criteria allowed other NSCLC histology than adenocarcinoma. Forty-five (88\%) patients had an ECOG PS score of 0 or 1 , while 6 (12\%) patients had an ECOG PS or 2 or 3. Thirty-two (63\%) had a 19del mutation, 13 (25\%) had L858R, and 6 (12\%) had minor EGFR mutations. Thirty-nine $(76 \%)$ patients received treatment with afatinib as a first-line EGFR-TKI setting (A group), and $12(26 \%)$ patients received afatinib as a second or

Table 1 Patient characteristics in a prospective observational study $(N=51)$

\begin{tabular}{lll}
\hline Characteristics & $\mathbf{N = 5 1}$ & \% \\
\hline Age, median (range) & $68(36-81)$ & \\
Sex (Male/Female) & $28 / 23$ & $55 / 45$ \\
Smoking (Yes/No) & $25 / 26$ & $49 / 51$ \\
EGFR mutation (19del/L858R/others) & $32 / 13 / 6$ & $63 / 25 / 22$ \\
$\begin{array}{l}\text { Stage at the diagnosis } \\
\text { (III/IV/postoperative recurence) }\end{array}$ & $1 / 45 / 5$ & $2 / 88 / 10$ \\
$\begin{array}{l}\text { Performance status } \\
\quad \text { 0-1/2 }\end{array}$ & \\
Treatment line with afatinib & $45 / 6$ & $88 / 12$ \\
$\quad$ First-line & & \\
$\quad$ Second or subsequent & 39 & 76 \\
\hline
\end{tabular}


subsequent EGFR-TKI therapy after gefitinib and/or erlotinib ( $\mathrm{G} / \mathrm{E} \rightarrow \mathrm{A}$ group).

\section{Detection of EGFR driver and T790M mutation in plasma in patients treated with afatinib}

Among 51 patients, 24 (47\%) and 5 (9.8\%) patients were positive for EGFR driver and T790M in plasma as assessed using the cobas test, respectively. In the A group (39 patients), 16 (41\%) patients were positive for a driver mutation and $3(8 \%)$ patients were positive for T790M in plasma using the cobas test at the time of progression. In the $\mathrm{G} / \mathrm{E} \rightarrow \mathrm{A}$ group (12 patients), 8 patients (67\%) were positive for a driver mutation and $2(17 \%)$ patients were positive for T790M in plasma. The detection rates for EGFR driver and T790M mutations in plasma in the A group were lower than the $\mathrm{G} / \mathrm{E} \rightarrow \mathrm{A}$ group, although the differences were not significant (Fig. 2a). Regarding the results of ddPCR, 19 (37\%) of the 51 patients had T790M mutation copies in plasma using ddPCR, and the T790M copy number ranged from 80 to 375,000 (Fig. 2b). Among these patients, 5 patients who had more than 400 copies/ $\mathrm{mL}$ also tested positive for the T790M mutation using the cobas test. In one patient who continued to receive afatinib treatment after RECIST-PD, blood samples were collected serially (Fig. 2c). The result of the first cfDNA analysis for T790M mutation using the cobas test was negative. However, the copy numbers for EGFR driver mutation and T790M increased as the site of metastasis progressed, and the second cfDNA analysis using cobas test resulted in a positive result for T790M mutation.

Regarding the concordance between the results of T790M mutation from plasma and tissue samples in patients with T790M mutation negative in plasma as assessed using the cobas test, 8 of the 14 patients with T790M copy positive in plasma using ddPCR underwent rebiopsy, and 3 of these patients had T790M mutation in tissue (Fig. 2d). In contrast, 20 of the 32 patients with T790M copy negative using ddPCR underwent rebiopsy, and 5 patients had T790M mutation in tissue. The presence of T790M mutation copy as detected using ddPCR did not influence the T790M mutation status in tissue obtained from patients with T790M negative results in plasma using the cobas test ( $37.5 \%$ vs. $40 \%, P=0.6508)$. In addition, we evaluated the PFS of initial-TKIs according to T790M mutation positivity (T790M copy: $\geq 400$ copies, $0<$ T790M copy: < 400, and T790M copy: negative). The copy number of T790M mutation as assessed using ddPCR did not affect the PFS (Supplemental Fig. 1).

\section{Detection rate of EGFR driver and T790M mutation between Gefitinib/Erlotinib and Afatinib groups}

Next, we evaluated whether the types of EGFR-TKIs (G/ $\mathrm{E}$ or afatinib) as first-line EGFR-TKI affected the detection of EGFR driver and T790M mutations in plasma.
Among the 51 patients who were enrolled in the prospective observational study, 29 patients who were treated with afatinib in a first-line setting and who had major EGFR mutations (Ex19 del and L858R) were selected (A arm). In addition, we retrospectively collected data from 33 patients who had been treated with only first-generation EGFR-TKIs (G/E) as a first-line setting and whose plasma samples had been assessed using the cobas test (G/E arm). The patient characteristics are shown in Table 2. The prevalences of males, smokers, and EGFR 19del were significantly higher in the A arm than in the G/E arm (males: $68.9 \%$ vs. $27.2 \%, P<0.01$; smoking: $44.8 \%$ vs. $24.2 \%, P=0.01$; EGFR 19 del: $79.3 \%$ vs. $51.5 \%, P=0.01)$.

Regarding the efficacies of EGFR-TKIs, no significant difference in PFS was observed between the A arm and the $\mathrm{G} / \mathrm{E}$ arm (16.4 vs. 13.5 months, $P=0.5580$ ) (Fig. 3a). Regarding the presence of EGFR-driver and T790M mutations in plasma, the detection rates for EGFR-driver and T790M mutation in plasma using the cobas test in patients treated with afatinib were lower than in those treated with $\mathrm{G} / \mathrm{E}$, although the differences were not significant (EGFR driver mutation: 34\% [A arm] vs. 52\% [G/E arm], $P=0.2072$; and T790M: $10 \%$ [A arm] vs. $27 \%$ [G/E arm], $P=0.1161$ ) (Fig. 3b). In addition, among patients who tested positive for the EGFR driver mutation in plasma, the detection rate for T790M mutation was not significant between the G/E and A arms (20\% vs. $52.9 \%, P=0.1241)$.

After initial EGFR-TKIs failure, 9 (31\%) patients in the A group and 15 (45\%) patients in the G/E group received osimertinib treatment. No significant difference in the PFS after osimertinib treatment was seen between the patients in the $A$ and G/E groups (9.0 months [G/E] vs. 9.0 months $[\mathrm{A}], P=0.7696$, Fig. 3c).

\section{Assessment of EGFR wild-type CNV in plasma}

In this study, we also analyzed the EGFR wild-type (wt) $\mathrm{CNV}$ in cfDNA in an exploratory manner using digital PCR. Twenty-three patients had a sufficient cfDNA volume for the assessment of EGFR-wt copy number. Two of the 23 patients had the T790M mutation in plasma as detected using the cobas test. An EGFR-wt copy number gain and loss were detected in each one patient (EGFR CNV: 1.28 and 23.9) (Fig. 4a and b). Interestingly, the patient who had a high EGFR CNV were treated with osimertinib after afatinib failure, but did not respond to the osimertinib treatment.

\section{Discussion}

In this study, we examined the detection rates for EGFR-driver and T790M mutations in plasma using the cobas test in EGFR-mutated NSCLC patients who had acquired resistance to afatinib in a real-world setting. 
A.

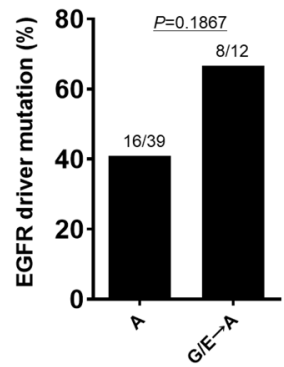

All patients $(\mathrm{N}=51)$

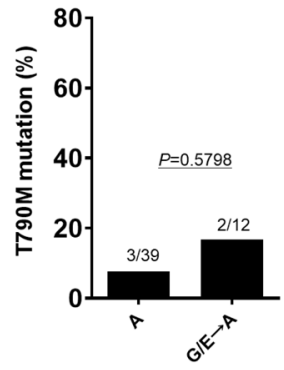

All patients $(\mathrm{N}=51)$

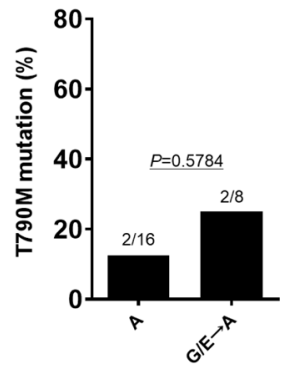

EGFR driver+ patients ( $\mathrm{N}=\mathbf{2 4})$
B.

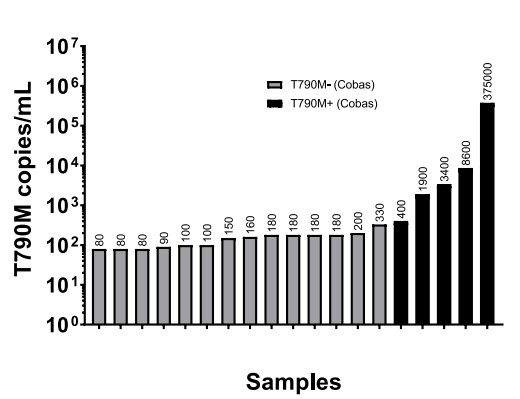

C.

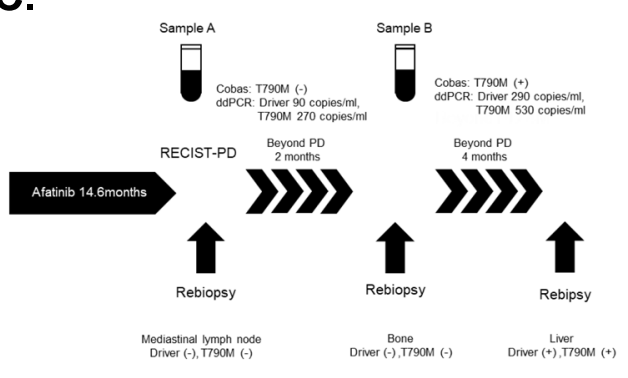

D.

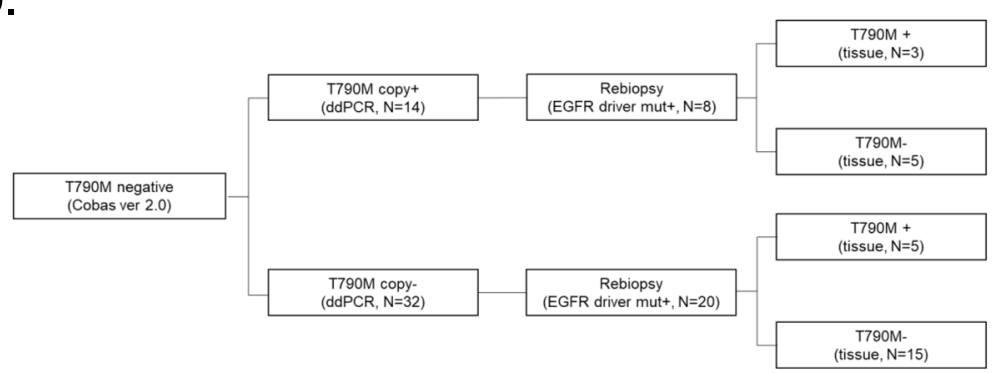

Fig. 2 Results of the prospective observational study. a Frequency of EGFR driver (left), T790M mutation in all patients (middle) and T790M mutation in EGFR driver mutation positive patients in plasma (right) according to the treatment line of afatinib (first-line vs. second or subsequent EGFR-TKIs setting after gefitinib and/or erlotinib). b T790M mutation copy numbers in plasma as assessed using ddPCR. c Serial analysis for T790M mutation in plasma obtained from a patient who continued afatinib treatment after RECIST-PD. d T790M mutation status in plasma as assessed using ddPCR and in tissue samples in patients with T790M negative results using the cobas test

Table 2 Patient Characteristics in the comparison study ( $N=62)$

\begin{tabular}{|c|c|c|c|}
\hline & Afatinib $(N=29)$. & $\mathrm{G} / \mathrm{E}(N=33)$. & $P$ value \\
\hline Age, median (range) & $66(36-80)$ & $68(41-80)$ & \\
\hline Sex (Male/Female) & $20 / 9$ & $9 / 24$ & 0.0010 \\
\hline Histology (Adenocarcinoma) & 29 & 33 & 1.0 \\
\hline Smoking (Yes/No) & $13 / 16$ & $8 / 25$ & 0.0126 \\
\hline Mutation (19del/ L858R) & $23 / 6$ & $17 / 16$ & 0.0225 \\
\hline Stage (III,IV/postoperative recurrence) & $25 / 4$ & $23 / 10$ & 0.1208 \\
\hline Treatment with Osimertinib (Yes/No) & $9 / 20$ & $15 / 18$ & 0.2448 \\
\hline
\end{tabular}


A.

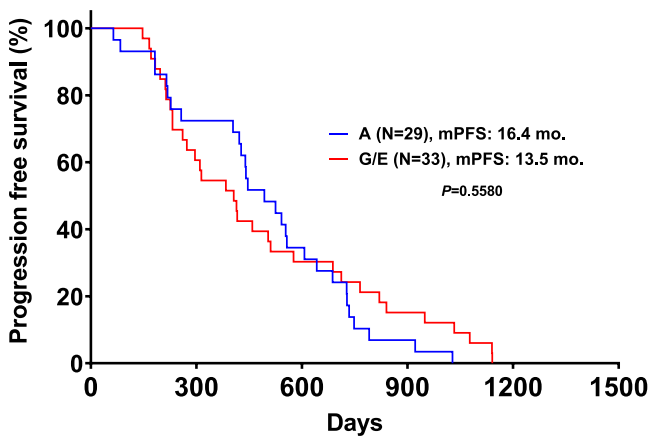

B.
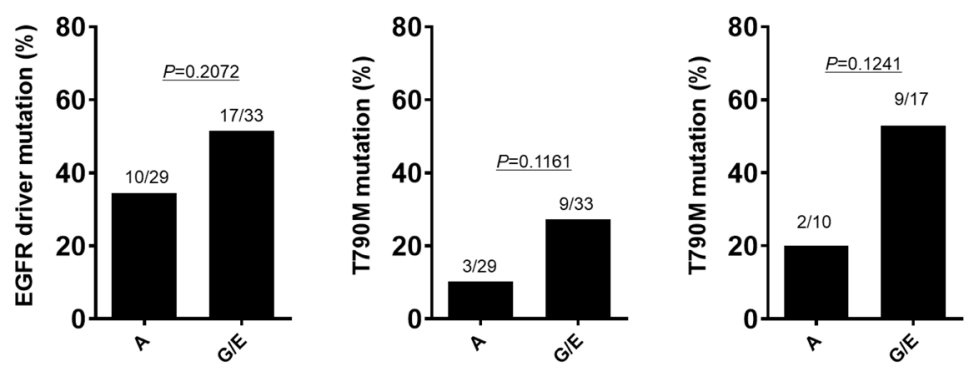

C.

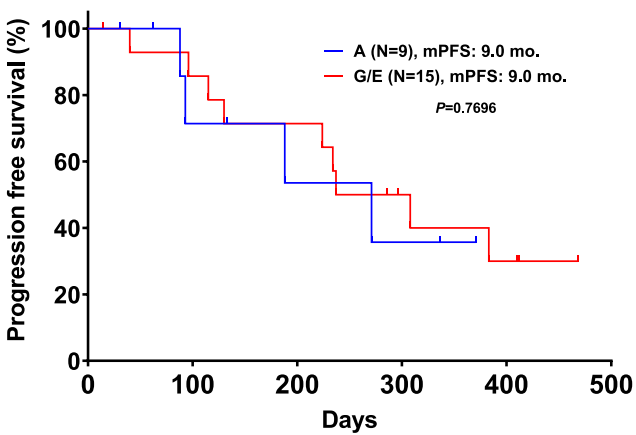

Fig. 3 Results of the comparison study. a PFS in patients treated with G/E or afatinib in a first-line EGFR-TKI setting. b Frequency of EGFR driver (left), T790M mutation in all patients (middle) and T790M mutation in EGFR driver mutation positive patients in plasma (right) according to the types of EGFR-TKIs (afatinib vs. gefitinib and/or erlotinib). c PFS of osimertinib after afatinib and G/E failure

The detection of T790M mutation in plasma using the cobas test was in complete agreement with the positive results (more than 400 copies $/ \mathrm{mL}$ ) for T790M mutation as assessed using ddPCR. In addition, this study also showed that the detection of EGFR driver and T790M mutations in plasma was lower in patients treated with first-line afatinib than in those with first-line G/E (34.5\% vs. $51.5 \%$ ), although the difference was not significant.

Some reports have shown that in $40-60 \%$ of patients with acquired resistance to afatinib, the emergence of T790M mutation was detected using tissue analyses, similar to the results for patients with acquired resistance to $G / E[12,13]$. However, Lee et al. recently reported that the occurrence of $\mathrm{T} 790 \mathrm{M}$ mutation in patients treated with afatinib is significantly lower than those with gefitinib and erlotinib [14]. In addition, Yoon et al. reported that although no significant difference was observed, the cumulative acquisition of the T790M mutation in patients treated with afatinib was lower than those with gefitinib $(48.8 \%$ vs. $59.3 \%, P=.317)$, since afatinib had a greater inhibitory activity, compared with G/ E, against EGFR-mutant cell lines harboring common mutations (including T790M) in vitro [15]. These results might be consistent with our data. Regarding the T790M status in plasma in patients treated with afatinib, Hochmair et al. reported that the T790M mutation was detected using a liquid biopsy in 47 out of 67 patients who had been treated with afatinib [16]. However, they 


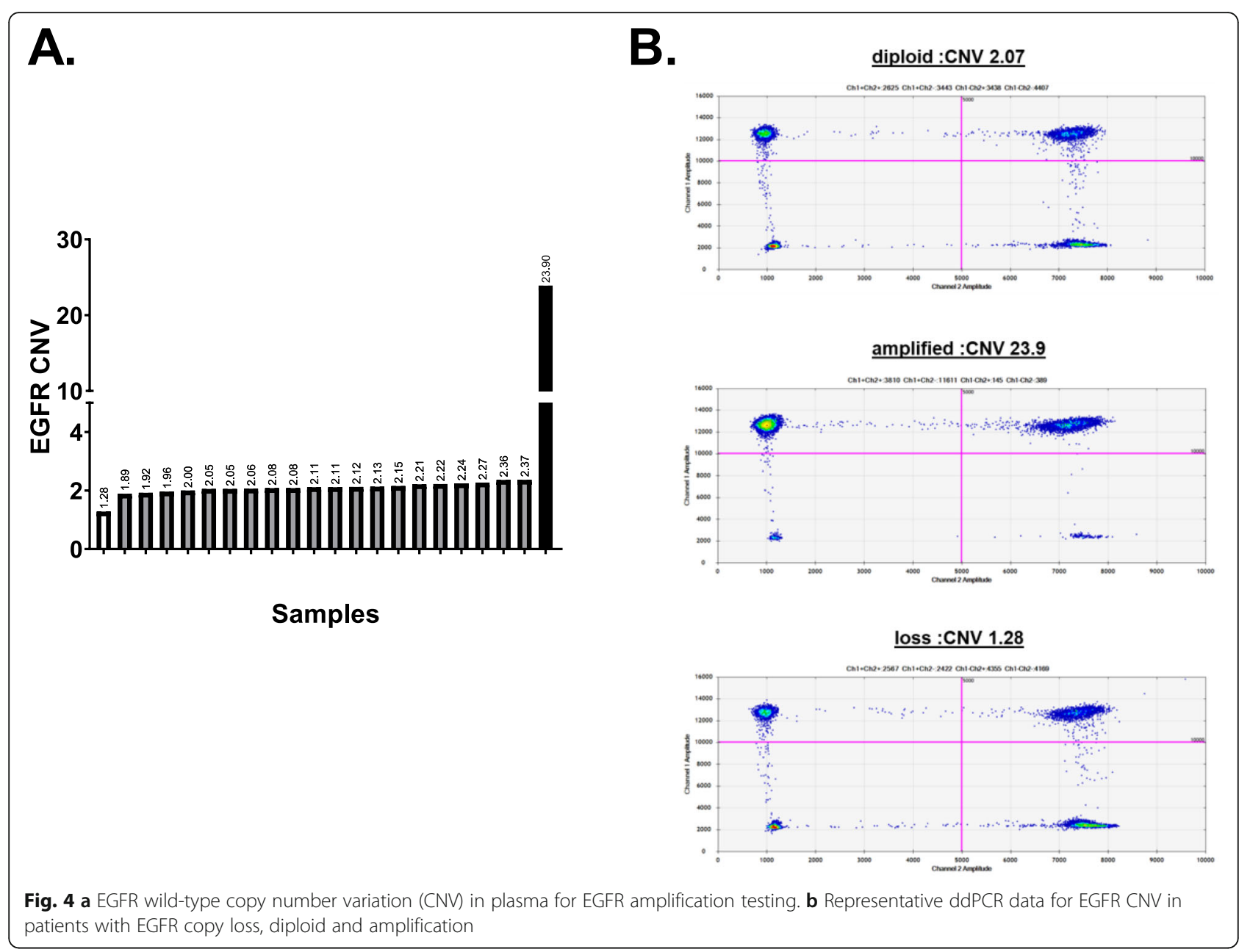

did not use the cobas test, but rather the ddPCR system, which is a more sensitive assay than the cobas test. In fact, in this study, among the 46 patients who tested negative for the T790M mutation in plasma using the cobas test, T790M mutation copies were detected using ddPCR in 14 patients. Therefore, the frequency of T790M mutation depends on the types of assays for T790M mutation.

The detection rates for EGFR driver and T790M mutations using the cobas test in our study were lower than those reported in the pooled AURA extension and AURA 2 data (EGFR driver mutations: $76-85 \%$ vs. $40-$ $51 \%$, T790M mutations: $61 \%$ vs. $5.7-27.2 \%$, respectively) [17]. One possible reason is the difference in terms of patient populations. The AURA studies included only patients with measurable disease to enroll the clinical trials. However, our study only included patients who acquired resistance to an initial TKI treatment, either afatinib or G/E. In addition, the positivity of EGFR driver and T790M mutations in plasma is related to the disease burden, and disease burden can affect positivity. Our data might be closer to the real-world setting for EGFR- mutated NSCLC. Additionally, Sacher et al. reported that when no sensitizing mutation is detected in patients with known EGFR-mutant lung cancer and acquired resistance, plasma genotyping for T790M can be uninformative [18]. To analyze the T790M mutation status in plasma more thoroughly, we evaluated the frequency of T790M mutation in EGFR driver mutation-positive NSCLC patients in plasma, but the detection of T790M mutation in EGFR driver mutation-positive patients was similar between the G/E and A arms.

Additionally, this study also showed that among 23 patients who had a sufficient cfDNA volume for assessment of EGFR wt copy number gain, one patient had EGFR wild-type amplification in cfDNA. EGFR wild-type amplification has also been reported as a mechanism of resistance to EGFR-TKIs, including osimertinib $[9,10]$. Indeed, the patient with EGFR wild-type amplification exhibited primary resistance to osimertinib.

The present study had several limitations. First, the sample size was relatively small. In addition, some of the patient characteristics, such as sex, smoking history and EGFR mutation type, differed between the $A$ and G/E 
arms in the comparison study. However, previous reports have shown that these characteristics did not affect the occurrence of T790M mutation after EGFR-TKIs. Second, the timing for re-biopsy using either tissue or liquid samples was not regulated. Furthermore, tissue analyses for EGFR T790M and EGFR amplification were not performed in all the patients at the same time as the plasma test.

\section{Conclusions}

The detection of EGFR-driver and T790M mutations in plasma using the cobas test might be lower in patients treated with afatinib than with gefitinib/erlotinib in a real-world setting. On the other hands, there was no significant differences of the detection of T790M mutations in tissue between two groups, and types of initial EGFRTKIs could affect the detection of EGFR-driver and T790M mutations in plasma at the progression. Further examination of the clinical utility of liquid biopsy, including the cobas test and ddPCR, for EGFR driver, T790M mutation and EGFR amplification is needed for the introduction of liquid biopsies into clinical practice in advanced EGFR-mutated NSCLC patients.

\section{Supplementary Information}

The online version contains supplementary material available at https://doi. org/10.1186/s12885-020-07777-2.

Additional file 1: Supplemental Fig. 1. PFS of initial-TKls according to T790M mutation positivity as assessed using ddPCR (T790M copy: $\geq 400$ copies vs. T790M copy: 0.01-400 vs. T790M copy: negative).

\section{Abbreviations \\ EGFR: Epidermal growth factor receptor; TKIs: tyrosine kinase inhibitors; NSCL C: non-small cell lung cancer; PFS: progression free survival; cfDNA: cell free DNA; RECIST: response evaluation criteria in solid tumours; ddPCR: Droplet Digital polymerase chain reaction; CNV: copy number variation; ECOG PS: Eastern Cooperative Oncology Group Performance Status; \\ PD: preogressive disease}

\section{Acknowledgements}

The authors would like to thank the following companies for their contributions: SRL Inc., Tokyo, Japan, and G\&G Science Inc., Fukushima, Japan for the analysis of cfDNA.

\section{Authors' contributions}

$\mathrm{YO}, \mathrm{TY}, \mathrm{Kl}$ and $\mathrm{TH}$ are conducting the study as described in the protocol and drafted this manuscript. YO, TY, KI and TH participated in the trial design and planning of the statistical analyses. YO and TY are conducting the translational research associated with this study. YO, TY, KA, TO, NI, SM, KI, TK, $E K, T M, A K, T K, T A, T$ and $T H$ are contributing the recruitment of participants and data collection and critically reviewed the manuscript. TY and TH are coprincipal investigator of the study. All authors approved the final version of the manuscript.

\section{Funding}

This research was supported by grants from Nippon Boehringer Ingelheim for the enrollment of patients and analyzing EGFR mutation in plasma, and the Aichi Cancer Research Foundation for the retrospective study.

\section{Availability of data and materials}

The datasets used and/or analyzed during the current study are available from the corresponding author on reasonable request.

\section{Ethics approval and consent to participate}

This study was conducted in accordance with the principles expressed in the Declaration of Helsinki. The full protocol was approved by the institutional review board in each institution. All participants in the study arm provided written informed consent before enrollment.

\section{Consent for publication}

Not applicable.

\section{Competing interests}

Dr. Yoshida has obtained research grants from Nippon Boehringer Ingelheim. Dr. Hida has obtained research grants from Novartis Pharma, Chugai Pharmaceutical, Taiho Pharmaceutical, AstraZeneca, Nippon Boehringer Ingelheim, Pfizer, Janssen Pharmaceutical, and Astellas, and has received personal fees from Novartis Pharma, Chugai Pharmaceutical, Taiho Pharmaceutical, AstraZeneca, Nippon Boehringer Ingelheim, and Pfizer. Dr. Ito has obtained personal fees from Nippon Boehringer Ingelheim, AstraZeneca, Pfizer, Eli Lilly, Chugai Pharmaceutical, MSD, Ono

Pharmaceutical, Taiho Pharmaceutical. All the other authors have no conflicts of interest. Dr. Ogutri has obtained research grant from Nippon Boehringer Ingelheim and speaker's bureau from Chugai Pharmaceutical, Taiho Pharmaceutical, Boehringer Ingelheim, AstraZeneca, Eli Lilly. Dr. Kimura has obtained speaker's bureau from Chugai Pharmaceutical, Taiho

Pharmaceutical, Boehringer Ingelheim, AstraZeneca, Novartis Pharma, Meiji Seika Pharmaceutical, MSD, Kyorin Pharmaceutical, Ono Pharmaceutical. Dr. Kubo has obtained honoraria from Boehringer Ingelheim.

\section{Author details}

1'Department of Thoracic Oncology, Aichi Cancer Center Hospital, 1-1 Kanokoden, Chikusa-ku, Nagoya, Aichi 464-8681, Japan. ${ }^{2}$ Current Address: Department of Thoracic Oncology, National Cancer Center Hospital, 5-1-1 Tsukiji, Chuo-ku, Tokyo 104-0045, Japan. ${ }^{3}$ Department of Respiratory Medicine, Shizuoka General Hospital, Shizuoka, Japan. ${ }^{4}$ Department of Respiratory Medicine, Allergy and Clinical Immunology, Nagoya City University, Nagoya, Japan. ${ }^{5}$ Second Division, Department of Internal Medicine, Hamamatsu University School of Medicine, Hamamatsu, Japan. ${ }^{6}$ Department of Respiratory Medicine, Fujita Health University School of Medicine, Toyoake, Japan. ${ }^{7}$ Respiratory Center, Matsusaka Municipal Hospital, Matsusaka, Japan. ${ }^{8}$ Department of Respiratory Medicine and Allergy, Tosei General Hospital, Seto, Japan. ${ }^{9}$ Department of Respiratory Medicine, Nagoya City West Medical Center, Nagoya, Japan. ${ }^{10}$ Department of Respiratory Medicine, Seirei Mikatahara General Hospital, Hamamatsu, Japan. ${ }^{11}$ Division of Respiratory Medicine and Allergology, Aichi Medical University School of Medicine, Nagakute, Japan. ${ }^{12}$ Department of Respiratory Medicine, National Hospital Organization Nagara Medical Center, Gifu, Japan. ${ }^{13}$ Department of Respiratory Medicine, Ogaki Municipal Hospital, Ogaki, Japan. ${ }^{14}$ Department of Respiratory Medicine, Toyama Prefectural Central Hospital, Toyama, Japan.

Received: 19 August 2020 Accepted: 28 December 2020

Published online: 12 January 2021

\section{References}

1. Mok TS, Wu YL, Thongprasert S, Yang CH, Chu DT, Saijo N, et al. Gefitinib or carboplatin-paclitaxel in pulmonary adenocarcinoma. N Engl J Med. 2009; 361(10):947-57.

2. Maemondo M, Inoue A, Kobayashi K, Sugawara S, Oizumi S, Isobe H, et al. Gefitinib or chemotherapy for non-small-cell lung cancer with mutated EGFR. N Engl J Med. 2010;362(25):2380-8.

3. Rosell R, Carcereny E, Gervais R, Vergnenegre A, Massuti B, Felip E, et al. Erlotinib versus standard chemotherapy as first-line treatment for European patients with advanced EGFR mutation-positive non-small-cell lung cancer (EURTAC): a multicentre, open-label, randomised phase 3 trial. Lancet Oncol. 2012;13(3):239-46.

4. Park K, Tan EH, O'Byrne K, Zhang L, Boyer M, Mok T, et al. Afatinib versus gefitinib as first-line treatment of patients with EGFR mutation-positive nonsmall-cell lung cancer (LUX-Lung 7): a phase 2B, open-label, randomised controlled trial. Lancet Oncol. 2016;17(5):577-89. 
5. Wu YL, Cheng Y, Zhou X, Lee KH, Nakagawa K, Niho S, et al. Dacomitinib versus gefitinib as first-line treatment for patients with EGFR-mutationpositive non-small-cell lung cancer (ARCHER 1050): a randomised, openlabel, phase 3 trial. Lancet Oncol. 2017:18(11):1454-66.

6. Yun $\mathrm{CH}$, Mengwasser KE, Toms AV, Woo MS, Greulich H, Wong KK, et al. The T790M mutation in EGFR kinase causes drug resistance by increasing the affinity for ATP. Proc Natl Acad Sci U S A. 2008;105(6):2070-5.

7. Oxnard GR, Arcila ME, Sima CS, Riely GJ, Chmielecki J, Kris MG, et al. Acquired resistance to EGFR tyrosine kinase inhibitors in EGFR-mutant lung cancer: distinct natural history of patients with tumors harboring the T790M mutation. Clin Cancer Res. 2011;17(6):1616-22.

8. Mok TS, Wu YL, Ahn MJ, Garassino MC, Kim HR, Ramalingam SS, et al. Osimertinib or Platinum-Pemetrexed in EGFR T790M-Positive Lung Cancer. N Engl J Med. 2017;376(7):629-40.

9. Nakatani K, Yamaoka T, Ohba M, Fujita Kl, Arata S, Kusumoto S, et al. KRAS and EGFR Amplifications Mediate Resistance to Rociletinib and Osimertinib in Acquired Afatinib-Resistant NSCLC Harboring Exon 19 Deletion/T790M in EGFR. Mol Cancer Ther. 2019;18(1):112-26.

10. Nukaga S, Yasuda H, Tsuchihara K, Hamamoto J, Masuzawa K, Kawada I, et al. Amplification of EGFR Wild-Type Alleles in Non-Small Cell Lung Cancer Cells Confers Acquired Resistance to Mutation-Selective EGFR Tyrosine Kinase Inhibitors. Cancer Res. 2017;77(8):2078-89.

11. Eisenhauer EA, Therasse P, Bogaerts J, Schwartz LH, Sargent D, Ford R, et al. New response evaluation criteria in solid tumours: revised RECIST guideline (version 1.1). Eur J Cancer. 2009;45(2):228-47.

12. Tanaka K, Nosaki K, Otsubo K, Azuma K, Sakata S, Ouchi H, et al. Acquisition of the T790M resistance mutation during afatinib treatment in EGFR tyrosine kinase inhibitor-naive patients with non-small cell lung cancer harboring EGFR mutations. Oncotarget. 2017;8(40):68123-30.

13. Nosaki K, Satouchi M, Kurata T, Yoshida T, Okamoto I, Katakami N, et al. Rebiopsy status among non-small cell lung cancer patients in Japan: A retrospective study. Lung Cancer. 2016;101:1-8.

14. Lee K, Kim Y, Jung HA, Lee SH, Ahn JS, Ahn MJ, et al. Repeat biopsy procedures and T790M rates after afatinib, gefitinib, or erlotinib therapy in patients with lung cancer. Lung Cancer. 2019;130:87-92.

15. Yoon BW, Kim JH, Lee SH, Choi CM, Rho JK, Yoon S, et al. Comparison of T790M Acquisition Between Patients Treated with Afatinib and Gefitinib as First-Line Therapy: Retrospective Propensity Score Matching Analysis. Translational Oncol. 2019;12(6):852-8.

16. Hochmair MJ, Buder A, Schwab S, Burghuber OC, Prosch H, Hilbe W, et al. Liquid-Biopsy-Based Identification of EGFR T790M Mutation-Mediated Resistance to Afatinib Treatment in Patients with Advanced EGFR MutationPositive NSCLC, and Subsequent Response to Osimertinib. Target Oncol. 2019:14(1):75-83.

17. Jenkins S, Yang JC, Ramalingam SS, Yu K, Patel S, Weston S, et al. Plasma ctDNA Analysis for Detection of the EGFR T790M Mutation in Patients with Advanced Non-Small Cell Lung Cancer. J Thorac Oncol. 2017;12(7):1061-70.

18. Sacher AG, Paweletz C, Dahlberg SE, Alden RS, O'Connell A, Feeney N, et al. Prospective Validation of Rapid Plasma Genotyping for the Detection of EGFR and KRAS Mutations in Advanced Lung Cancer. JAMA Oncol. 2016; 2(8):1014-22.

\section{Publisher's Note}

Springer Nature remains neutral with regard to jurisdictional claims in published maps and institutional affiliations.

\section{Ready to submit your research? Choose BMC and benefit from:}

- fast, convenient online submission

- thorough peer review by experienced researchers in your field

- rapid publication on acceptance

- support for research data, including large and complex data types

- gold Open Access which fosters wider collaboration and increased citations

- maximum visibility for your research: over $100 \mathrm{M}$ website views per year

At BMC, research is always in progress.

Learn more biomedcentral.com/submissions 